\title{
Cytokine and chemokine profiles in fibromyalgia, rheumatoid arthritis and systemic lupus erythematosus: a potentially useful tool in differential diagnosis
}

\author{
Daniel J. Wallace • Igor M. Gavin • Oleksly Karpenko • \\ Farnaz Barkhordar · Bruce S. Gillis
}

Received: 26 July 2014 / Accepted: 28 October 2014 / Published online: 7 November 2014

(C) The Author(s) 2014. This article is published with open access at Springerlink.com

\begin{abstract}
Making a correct diagnosis is pivotal in the practice of clinical rheumatology. Occasionally, the consultation fails to provide desired clarity in making labeling an individual as having fibromyalgia (FM), systemic lupus erythematosus (SLE) or rheumatoid arthritis (RA). A chemokine and cytokine multiplex assay was developed and tested with the goal of improving and achieving an accurate differential diagnosis. 160 patients with FM, 98 with RA and 100 with SLE fulfilling accepted criteria were recruited and compared to 119 controls. Supernatant cytokine concentrations for IL-6, IL-8, MIP-1 alpha and MIP-1 beta were determined using the Luminex multiplex immunoassay bead array technology after mitogenic stimulation of cultured peripheral blood mononuclear cells. Each patient's profile was scored using a logistical regression model to achieve statistically determined weighting for each chemokine and cytokine. Among the 477 patients evaluated, the mean scores for FM $(1.7 \pm 1.2$; $1.52-1.89)$, controls $(-3.56 \pm 5.7 ;-4.59$ to -2.54$)$, RA $(-0.68 \pm 2.26 ;-1.12$ to -0.23$)$ and SLE $(-1.45 \pm 3.34$, -2.1 to -0.79$)$. Ninety-three percent with FM scored
\end{abstract}

\section{J. Wallace $(\bowtie)$}

Wallace Rheumatic Diseases Study Center, West Hollywood, CA, USA

e-mail: dwallace@ucla.edu

I. M. Gavin · O. Karpenko · F. Barkhordar · B. S. Gillis

EpicGenetics, Los Angeles, CA, USA

\section{M. Gavin}

Research Resources Center, University of Illinois at Chicago,

Chicago, IL, USA

B. S. Gillis

College of Medicine, University of Illinois at Chicago, Chicago, IL, USA positive compared to only $11 \%$ of healthy controls, $69 \%$ RA or $71 \%$ SLE patients had negative scores. The sensitivity, specificity, positive predictive and negative predictive value for having FM compared to controls was 93, 89, 92 and $91 \%$, respectively $\left(p<2.2 \times 10^{-16}\right)$. Evaluating cytokine and chemokine profiles in stimulated cells reveals patterns that are uniquely present in patients with FM. This assay can be a useful tool in assisting clinicians in differentiating systemic inflammatory autoimmune processes from FM and its related syndromes and healthy individuals.

Keywords Fibromyalgia - Rheumatoid arthritis . Systemic lupus erythematosus - Cytokines - Chemokines · Peripheral blood mononuclear cells · Phytohemagglutinin

\section{Introduction}

The differential diagnosis of rheumatic disorders involves distinguishing inflammation, degenerative changes and central sensitization from each other. Making this distinction is usually obvious or easily discernible on the basis of taking a history, performing a physical examination, viewing imaging studies as appropriate and obtaining laboratory analyses. There are occasions where traditional methods fail to either elicit a diagnosis or give the clinician clarity with regards to whether a patient, for example, is inflamed or has fibromyalgia (FM) [1].

Fibromyalgia has been a medical condition that for decades was relegated to being defined as a collection of subjective symptoms of varying characteristics lacking objective and reproducible laboratory features [2]. FM is estimated to afflict a population in the USA alone of between 2 and $5 \%$ of the US population [3]. The annual cost of diagnosing FM makes it one of the most expensive 
Table 1 Characteristics of patients

\begin{tabular}{|c|c|c|c|c|}
\hline & Control & FM & RA & SLE \\
\hline Cohort size & 119 & 160 & 98 & 100 \\
\hline \multicolumn{5}{|l|}{ Age } \\
\hline Range & $18-76$ & $18-82$ & $21-92$ & 21-92 \\
\hline Median & 41 & 53.2 & 59 & 48 \\
\hline Mean & 40.8 & 52.2 & 58.1 & 49.6 \\
\hline \multicolumn{5}{|l|}{ Sex } \\
\hline Female & 101 & 98 & 86 & 94 \\
\hline Male & 18 & 12 & 12 & 6 \\
\hline
\end{tabular}

diagnostic processes linked to any illness [4] as it presently chiefly relies on using tests to "rule out" disorders of rheumatic, neurologic, psychiatric, hematologic and endocrine origins. Hence, finding an objective diagnostic methodology offers substantial medical cost benefits. It additionally could lead to a potential way to monitor treatment efficacy as well as eliminate what has been an inexorable and prolonged pathway to an endpoint diagnosis that has previously utilized an indirect process of elimination.

In the last few years, laboratory panels have demonstrated that the degree or intensity of inflammation, for instance, may be further elucidated by composite scores or weighted testing that are statistically significant (e.g., multibiomarker disease activity <Vectra $>$ for RA, cellbound complement activation products <Avise $>$ for SLE) $[5,6]$. The multibiomarker disease activity index used in RA, for example, includes cytokine levels in its weighted scoring. In 2012, a group at the University of Illinois College of Medicine evaluated 8 cytokines and chemokines in 110 FM patients and 91 matched healthy controls [7]. Unlike most previous studies, rather than measuring serum levels, peripheral blood mononuclear cells (PBMC) were stimulated by mitogens and in vitro production was quantitated. Statistically, significant findings distinguished the two groups as the FM group had 1.4-8 fold decreases in cytokine/chemokine production. Post hoc analyses determined that the panel was just as accurate if four chemokines and cytokines (instead of eight) were studied. Since FM is common in patients with autoimmune disorders, such as systemic lupus erythematosus (SLE) [8, 9], Sjogren's Syndrome [10,11] and rheumatoid arthritis (RA) [12], the chemokine/cytokine patterns found in FM patients may not have been unique to FM and were perhaps merely linked to rheumatic disorders as a whole, especially because of the frequent coexistence of FM and RA and FM and SLE. We therefore undertook comparative analyses to test the hypothesis that inflammation (represented by RA and SLE) and central sensitization syndromes (represented by FM) have distinct cytokine/chemokine signatures that could be clinically relevant.

\section{Methods}

A total of $160 \mathrm{FM}$ patients diagnosed with FM for at least 1 year were recruited to the study. Patients underwent two separate and independent physical examinations by two physicians to confirm that they met the American College of Rheumatology criteria [2]. All FM patients had discontinued their FM-related medications for 2 weeks prior to testing. One hundred SLE and 98 RA patients treated in University of Illinois College of Medicine were also studied. All fulfilled American College of Rheumatology criteria $[13,14]$. Information regarding their demographics, disease duration and concurrent medication was collected (Table 1). Patients with SLE or RA who also had FM were excluded. The healthy controls were age- and sex-matched to the FM patients only and lacked a history of any type of chronic or acute illnesses and none were using any medications, over the counter or prescription drugs that had any known immunologic effects. After obtaining IRB approved informed consent, PBMC were obtained from each patient as described earlier [7]. Concentrations of live cells as well as percentages of dead cells in PBMC fractions were measured by using an automatic cell counter (Cellometer Auto 2000, Naxcelom Biosciences) according to the manufacturer's instructions. These cells were cultured in the presence of the mitogenic activator phytohemagglutinin (PHA). After a culture time of $24 \mathrm{~h}$, the resultant supernatant was harvested. Supernatant cytokine concentrations of interleukin-6, interleukin-8, macrophage inflammatory protein- 1 alpha and macrophage inflammatory protein-beta (IL-6, IL-8, MIP-1a and MIP-1b) were determined using the Luminex multiplex immunoassay bead array technology [7]. Serial dilutions of cytokine standards were run in duplicates in each assay; their readings were used for calculating standard curves. In addition, pooled culture supernatants obtained from activated cell cultures served as a positive control. Fluorescence was measured using a MagPix fluorescence bead reader (Luminex).

Statistical analyses

Fluorescence intensities were transferred into $\mathrm{R}$ (http:www.r-project.org) statistical software [15] for converting into concentration values. The standard curve was fitted with a 5-PL model, and the concentrations of the cytokines were quantified according to the curve. A twosided $t$ test with unequal variance was used to test whether the mean concentrations of each cytokine were the same in the groups. The descriptive statistics of the groups as well as the $p$ values of the $t$ test were calculated by using the stats package for $\mathrm{R}$.

We used function $1 \mathrm{rm} *$ from package rms [16] in $\mathrm{R}$ software to determine a logistical regression model on the concentration of the cytokines described above as predictor 
Table 2 Cytokine/chemokine composite (CCC) test scores of patients and healthy controls

\begin{tabular}{|c|c|c|c|c|c|c|}
\hline & Subjects tested & Mean score & $95 \%$ Confidence interval & $\mathrm{CCC}$ test score & $\%$ Positive & $\%$ Negative \\
\hline Healthy controls & 119 & $-3.56 \pm 5.7$ & -4.59 to -2.54 & 2.8 & $13(11 \%)$ & $106(89 \%)$ \\
\hline FM & 160 & $1.7 \pm 1.2$ & 1.52 to 1.89 & 84.6 & $149(93 \%)$ & $11(7 \%)$ \\
\hline RA & 98 & $-0.68 \pm 2.26$ & -1.12 to -0.23 & 33.7 & $30(31 \%)$ & $68(69 \%)$ \\
\hline SLE & 100 & $1.45 \pm 3.34$ & -2.1 to -0.79 & 19 & $29(29 \%)$ & $71(71 \%)$ \\
\hline
\end{tabular}

Table 3 Characteristics of the CCC test scores in FM versus controls

\begin{tabular}{ll}
\hline Sensitivity & $93 \%$ \\
Specificity & $89 \%$ \\
Positive predictive value & $92 \%$ \\
Negative predictive value & $91 \%$ \\
Area under ROC curve & 0.96 \\
Wilcoxon test for FM scores versus controls & $p<2.2 \times 10^{-16}$ \\
\hline
\end{tabular}

variables, and binary dependent variables and test scores were calculated.

The measured concentrations of the four cytokines, $c_{\mathrm{IL}-6}, c_{\mathrm{IL}-8}, c_{\mathrm{MIP}-1 \mathrm{a} \text { and }} c_{\mathrm{MIP}-1 \mathrm{~b}}$, are combined into a single score using the following formula: $x=$ intercept + $w_{\text {IL-6 }} c_{\text {IL-6 }}+w_{\text {IL-8 }} c_{\text {IL-8 }}+w_{\text {MIP-1a }} c_{\text {MIP-1a }}+w_{\text {MIP-1b }} c_{\text {MIP-1b }}$. Weights $w_{\mathrm{IL}-6}, w_{\mathrm{IL}-8}, w_{\mathrm{MIP}-1 \mathrm{a}}$ and $w_{\mathrm{MIP}-1 \mathrm{~b}}$, and intercept were determined experimentally by fitting a logistic regression curve. In addition, a cytokine/chemokine composite test score on the scale of 0-100 was calculated using the following formula:

Cytokine/chemokine composite test score $=\frac{1}{1+e^{-x}} * 100$.

\section{Results}

Fibromyalgia patients clearly had higher scores; the mean score was $1.7 \pm 1.2$, with $1.52-1.89$ confidence interval which corresponds to 84.6 value of cytokine/chemokine test score on the scale of 0-100 (Table 2). The mean score of the control patients was $-3.56 \pm 5.7$, with -4.59 to -2.54 confidence interval (cytokine/chemokine composite $<\mathrm{CCC}>$ test score 2.8). If a "profile" meant having a positive score (or greater than 50 on a scale of 100 for CCC test score), $93 \%$ with FM and only $11 \%$ of healthy controls met this benchmark. This was considered to be $93 \%$ sensitive and $89.4 \%$ specific for the diagnosis of FM when compared to the 119 controls (Table 3). The Wilcoxon test for FM versus healthy controls was $p<2.2 \times 10^{-16}$, and the area under the receiver operating characteristic (ROC) curve was 0.96 (Fig. 1). Following completion of this part of the protocol, 100 patients with SLE and 98 RA patients were studied. A total of $71 \%$ of those with SLE and $69 \%$ with RA had negative

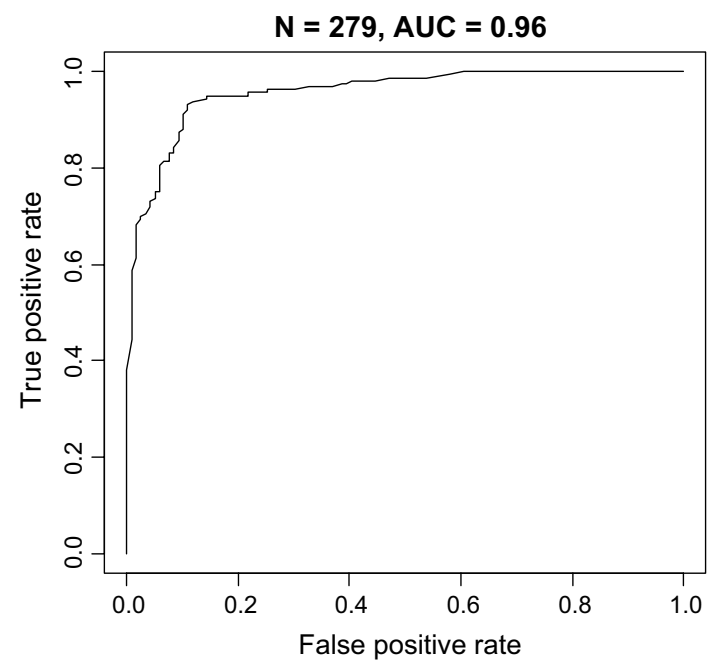

Fig. 1 Receiver operating characteristic (ROC) curve for CCC test scores of $160 \mathrm{FM}$ patients and 119 controls. The area under the curve (AUC) is 0.96

scores $(<50$ on a scale of 100$)$. The mean scores were $-0.68 \pm 2.26$ for RA patients $(-1.12$ to -0.23 confidence interval) and $-1.45 \pm 3.34$ for SLE patients $(-2.1$ to -0.79 confidence interval). Corresponding cytokine/ chemokine composite test scores were 33.7 and 19 on a scale of 100 .

To better understand the statistical distribution of cytokine profile scores, each patient's test score was calculated as a percentile rounded to the nearest integer on a scale of 1-100. There were two peaks in the scoring distribution of the RA and SLE patients: one in the low score region (48\% with SLE and $42 \%$ with RA for example had scores between 0 and 29) and a second smaller peak in the highest scoring region (25\% with SLE and $28 \%$ with RA had scores between 70 and 100) (Fig. 2). This suggests that immune modulating agents, corticosteroids or concurrent FM in the autoimmune patients may account for the findings. Nevertheless, the SLE/RA versus FM scores achieved a significance of $p<2.2 \times 10^{-16}$ as calculated by the Wilcoxon rank sum test. Concentrations of the studied cytokines in PBMC culture supernatants tended to be lower in FM patients compared to the autoimmune subjects, except for RA 


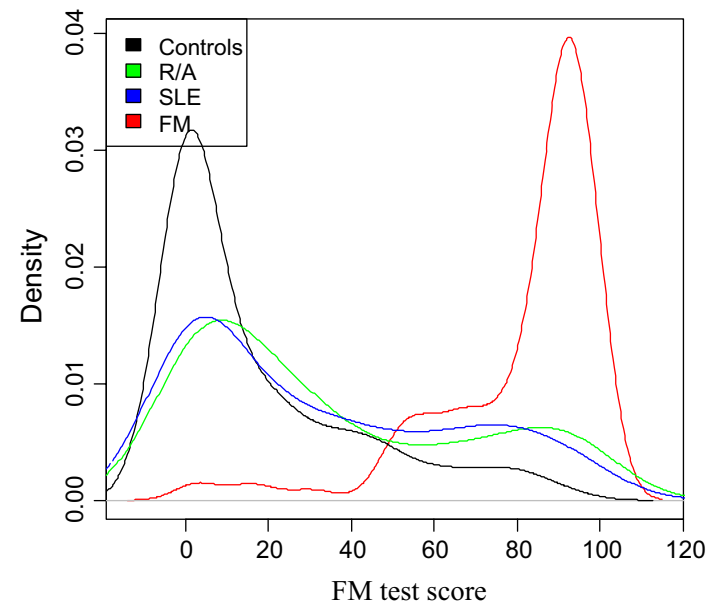

Fig. 2 The probability densities of CCC test scores of 119 controls, $160 \mathrm{FM}$ patients, 100 SLE patients and 98 RA patients

where IL-8 concentrations were similar (Table 4). Interestingly, FM patients showed the lowest levels of IL-6 compared to RA, SLE patients and controls-which were within $2 \%$ of each other $(p<0.00001)$. Most of the autoimmune patients disease was under good control with medication.

\section{Discussion}

Nearly 500 FM, autoimmune and healthy control patients underwent testing for cytokine/chemokine activity after mitogenic stimulation. Using a numerical score, all three groups had unique patterns with FM patients demonstrating less response to stimulation. This cytokine profile test had a $93 \%$ sensitivity and an $89.4 \%$ specificity for the diagnosis of FM. We also found that these profiles are relatively sensitive and specific for FM compared to SLE and RA. It remains unclear if these differences are directly related to the pathogenesis of FM.

We have been studying the role of cytokines in fibromyalgia for 30 years $[17,18]$. Tumor necrosis factor and interleukins $1,2,6,8$ and 10 are associated with pain modulation, sleep induction, cognitive dysfunction, antinociception and sympathetic nervous system homeostasis to varying degrees. Studies assessing serum levels in our opinion are unreliable due to short half-lives, circadian rhythms (time of day that the measurements are obtained) as well as target tissue variance. Published reports have not demonstrated a consistent FM pattern. A few studies have looked at cytokine function after mitogenic stimulation. Patients with self-reported FM of $<2$ years duration had a greater response to stimulation than those with chronic FM [17]. This is consistent with findings from the

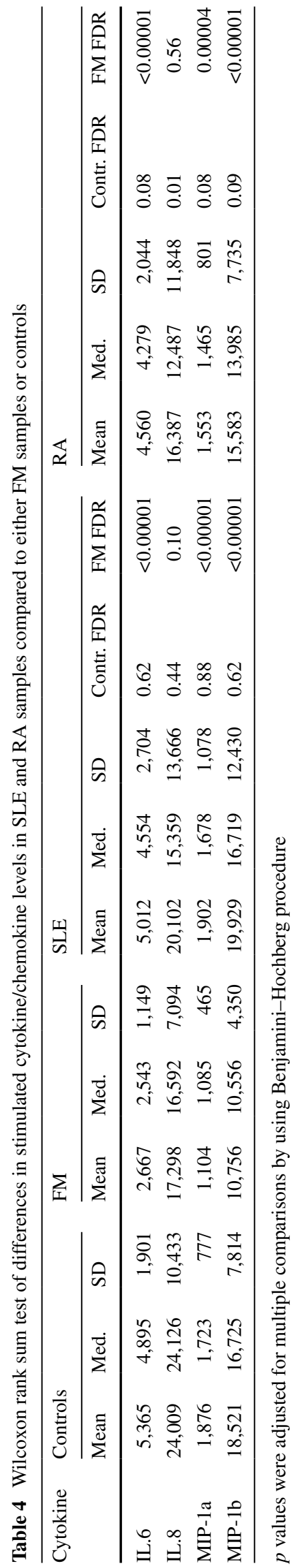


National Institutes of Health that although serum cortisol levels are normal in the syndrome, response to cortrosyn stimulation is blunted [18]. Further work has suggested that sympathetic nervous system responses are less robust in FM when ascertained by decreased heart rate variability or exercise $[19,20]$.

The scores found in this report are consistent with the finding that chronic stress decreases cytokine/chemokine responses. IL-6 is an acute phase reactant that is associated with stress, fatigue, hyperalgesia and sympathetic nervous system activation; IL-8 induces chemotaxis, phagocytosis, angiogenesis and modulates sympathetic mediated pain, and MIP-1 alpha and beta recruit polymorphonuclear cells and are chemoattractants for natural killer cells and monocytes. In all probability, chronic sensitization syndromes (which includes FM) are associated with a milieu whereby responses to sympathetic, hormonal, cytokine and chemokine stimulation are diminished. In the last decade, it has also become evident that glial cells produce cytokines, and complex interactions in the setting of FM might explain opioid induced hyperalgesia observed in the syndrome [21]. In other words, FM patients often fare worse when prescribed narcotic analgesics.

The expression of serum cytokines and chemokines in RA and SLE is increased, and the use of anticytokine therapies (and Phase 1 and 2 studies of chemokine inhibition) for autoimmune disease suggests that the findings would be opposite of what has been shown in FM [22].

Our study was confounded by different times of day that the cytokine/chemokine profile was obtained, concomitant anti-inflammatory and immune modulating medications, the use of corticosteroids and coexistence of FM in $<10 \%$ of the autoimmune (SLE and RA) patients. Despite this, we were able to demonstrate statistically significant differences in scores comparing patients with FM, healthy controls and autoimmune disease. The study was exploratory and provided preliminary information documenting a signal. Now that there is one, further data mining and/or a more focused second study would allow us to examine which subsets or autoimmune "phenotypes" are more likely to influence a combined chemokine score. This would, for example, include looking at established disease activity indices and acute phase reactants (e.g., sedimentation rate, $\mathrm{C}$ reactive protein). High cytokine/chemokine composite test scores strongly suggest a central sensitization component with or without an inflammatory condition. We posit that using a cytokine/chemokine stimulated response composite score is clinically useful in the differential diagnosis of FM patients as well as in patients where the role of inflammation versus central sensitization would benefit from further delineation. For example, changes in the score could be used to monitor response to different interventions.
Acknowledgments Dr. Gillis personally underwrote the study and owns EpicGenetics. Dr. Wallace was a consultant for EpicGenetics in 2013. Dr. Gavin, Karpenko and Barkhordar are employees of EpicGenetics.

Open Access This article is distributed under the terms of the Creative Commons Attribution License which permits any use, distribution, and reproduction in any medium, provided the original author(s) and the source are credited.

\section{References}

1. Wallace DJ, Schwartz E, Chi-Lin H, Peter JB (1995) The 'rule out lupus' rheumatology consultation: clinical outcomes and perspectives. J Clin Rheumatol 1(3):158-164

2. Wolfe F, Smythe HA, Yunus MB, Bennett RM, Bombardier C, Goldenberg DL et al (1990) The American College of Rheumatology 1990 criteria for the classification of fibromyalgia, report of the multicenter criteria committee. Arthritis Rheum 33:160-172

3. Clauw DJ, Fibromyalgia A (2014) Clinical review. JAMA 311: $1547-1555$

4. Chandran A, Schaefer C, Ryan K, Baik R, Mc Nett M, Zlateva G (2012) The comparative economic burden of mild, moderate, and severe fibromyalgia: results from a retrospective chart review and cross-sectional survey of working-age US adults. J Manag Care Pharm 18:415-426

5. Curtis JR, n Mil AH, Knevel R, Huizinga TW, Haney DJ, Shen $\mathrm{Y}$ et al (2012) Validation of a novel multibiomarker test to assess rheumatoid arthritis disease activity. Arthritis Care Res (Hoboken) 64:1794-1803

6. Kalunian KC, Chatham WW, Massarotti EM, Reyes-Thomas J, Harris C, Furie RA (2012) Measurement of cell-bound complement activation products enhances diagnosic performance in systemic lupus erythematosus. Arthritis Rheum 64(12):4040-4047. doi:10.1002/art.34669

7. Behm FG, Gavin IM, Karpenko O, Lindgren V, Gaitonde S, Gashkoff PA, Gillis BS (2012) Unique immunologic patterns in fibromyalgia. BMC Clin Pathol 12:25. doi:10.1186/1472-6890-12-25

8. Middleton GD, McFarlin JE, Lipsky PE (1994) The prevalence and clinical impact of fibromyalgia in systemic lupus erythematosus. Arthritis Rheum 37:1181-1188

9. Morand EF, Miller MH, Whittingham S, Littlejohn GO (1994) Fibromyalgia syndrome and disease activity in systemic lupus erythematosus. Lupus 3:187-191

10. Vitali C, Tavoni A, Neri R, Castrogiavanni P, Bombardieri S, Pasero G et al (1989) Fibromyalgia features in patients with primary Sjogren's syndrome. Evidence of a relationship with psychological depression. Scand J Rheumatol 18:21-27

11. Tishler M, Barak Y, Paran D, Yaron M (1997) Sleep disturbances, fibromyalgia and primary Sjogren's syndrome. Clin Exp Rheumatol 15:71-74

12. Wolfe F, Michaud K (2004) Severe rheumatoid arthritis (RA), worse outcomes, comorbid illness, and sociodemographic disadvantage characterize RA patients with fibromyalgia. J Rheumatol 31:695-700

13. Hochberg MC (1997) Updating the American College of Rheumatology revised criteria for the classification of systemic lupus erythematosus. Arthritis Rheum 40:1725

14. Aletaha D, Neogi T, Silman AJ, Funovits J, Felson DT, Bingham CO III (2010) 2010 rheumatoid arthritis classification criteria. An American College of Rheumatology/European League Against Rheumatism collaborative initiative. Arthritis Rheum 62:2569-2581 
15. R Core Team (2013) A language and environment for statistical computing. Vienna, Austria, http://www.R-project.org/

16. Harrell FE (2013) RMS: regression modeling strategies. http:// CRAN-R-project.org/package $=\mathrm{rms}$

17. Wallace DJ, Linker-Israeli M, Hallegua D, Silverman S, Silver D, Weisman MH (2001) Cytokines play an aetiopathogenetic role in fibromyalgia: a hypothesis and pilot study. Rheumatology 40:743-749

18. Wallace DJ, Margolin K, Waller P (1988) Fibromyalgia and interleukin therapy for malignancy. Ann Intern Med 108:909

19. Crofford LJ, Pillemer SR, Kalogeras KT, Cash JM, Michelson D, Kling MA et al (1994) Hypothalamic-pituitary-adrenal axis pertubations in patients with fibromyalgia. Arthritis Rheum 37(11):1583-1592
20. Meeus M, Goubert D, De Backer F, Struyf F, Hermans L, Coppieters I et al (2013) Heart rate variability in patients with fibromyalgia and patients with chronic fatigue syndrome: a systemicatic review. Semin Arthritis Rheum 43(2):279-287. doi:10.1016/ j.semarthrit.2013.03.004 (Epub 2013 Jul 6). Review

21. Watkins LR, Hutchinson MR, Rice KC, Maier SF (2009) The "toll" of opioid-induced glial activation: improving the clinical efficacy of opioids by targeting glia. Trends Pharmacol Sci 30(11):581-591. doi:10.1016/j.tips.2009.08.002 (Epub 2009 Sep 15)

22. Grondal G, Gunnarsson I, Ronnelid J, Rogberg S, Klareskog L, Lundberg I (2000) Cytokine production, serum levels and disease activity in systemic lupus ertythematosus. Clin Exp Rheumatol 18:565-570 